\title{
Retrolabyrinthine approach for cochlear nerve preservation in neurofibromatosis type 2 and simultaneous cochlear implantation
}

\author{
Ricardo Ferreira Bento ${ }^{1}$, Tatiana Alves Monteiro², Aline Gomes Bittencourt ${ }^{3}$, Maria Valeria Schmidt Goffi-Gomez', \\ Rubens de Brito5.
}

1) Otolaryngologist, PhD. Professor and Chairman. Department of Otolaryngology, University of São Paulo School of Medicine, São Paulo, Brazil.

2) Otolaryngologist. Department of Otolaryngology, University of São Paulo School of Medicine, São Paulo, Brazil.

3) Otolaryngologist, PhD student. Neurotology Fellow. Division of Otorhinolaryngology, University of São Paulo Medical School, São Paulo, Brazil.

4) Audiologist, PhD in Human Communication Disorders (Speech Pathology). Department of Otolaryngology, University of São Paulo School of Medicine, São Paulo, Brazil.

5) Otolaryngologist, PhD. Associate Professor. Department of Otolaryngology, University of São Paulo School of Medicine, São Paulo, Brazil.

Institution: Division of Otorhinolaryngology, University of São Paulo Medical School

São Paulo/SP - Brazil.

Mailing address: Dra. Aline Gomes Bittencourt - Departamento de Otorrinolaringologia - Hospital das Clínicas - Universidade de São Paulo - Av. Dr. Enéas de Carvalho Aguiar, 255, 6andar/sala 6167 - São Paulo / SP - Brazil - Zip code: 05403-000 Telephone: (+ 55 11) 3088-0299 - E-mail: alinebittencourt@hotmail.com

Article received on March 18 ${ }^{\text {th }}, 2013$. Article accepted on April 18 ${ }^{\text {th }}, 2013$.

\section{SUMMARY}

Introduction: Few cases of cochlear implantation (CI) in neurofibromatosis type 2 (NF2) patients had been reported in the literature. The approaches described were translabyrinthine, retrosigmoid or middle cranial fossa.

Objectives: To describe a case of a NF2- deafened-patient who underwent to vestibular schwannoma resection via RLA with cochlear nerve preservation and CI through the round window, at the same surgical time.

Resumed Report: A 36-year-old woman with severe bilateral hearing loss due to NF2 was submitted to vestibular schwannoma resection and simultaneous CI. Functional assessment of cochlear nerve was performed by electrical promontory stimulation. Complete tumor removal was accomplishment via RLA with anatomic and functional cochlear and facial nerve preservation. Cochlear electrode array was partially inserted via round window. Sound field hearing threshold improvement was achieved. Mean tonal threshold was $46.2 \mathrm{~dB}$ HL. The patient could only detect environmental sounds and human voice but cannot discriminate vowels, words nor do sentences at 2 years of follow-up.

Conclusion: Cochlear implantation is a feasible auditory restoration option in NF2 when cochlear anatomic and functional nerve preservation is achieved. The RLA is adequate for this purpose and features as an option for hearing preservation in NF2 patients.

Keywords: Neurofibromatosis 2; Cochlear Implantation; Hearing Loss.

\section{INTRODUCTION}

Neurofibromatosis type $2(\mathrm{NF} 2)$ is an autosomal dominant disease consequent to mutation of both alleles of a suppressor tumor gene in the long arm of chromosome $22(1,2)$. Bilateral vestibular schwannoma is typical, occurring in $90 \%$ of the gene carriers (1). Progressive sensorineural hearing loss is almost certain, accompanied or not with dizziness and tinnitus.

The first attempt at auditory restoration in a patient with NF2 and profound hearing loss was conducted by House and Hitselberger in 1979 (3). Auditory brainstem implant (ABI) has been the standard surgical treatment for these patients, to recover some degree of auditory capability and to enhance lip reading. In the 1990s, the promising idea of using a cochlear implant for auditory restoration became feasible. Auditory results of cochlear implantation are far better than those reported after ABI (4).
The aim of this study is to describe a ase of a NF2deafened-patient whounderwent tovestibular schwannoma resection via retrolabyrinthine approach, with cochlear nerve preservation and cochlear implantation through the round window, at the same surgical time.

\section{Case Report}

A 36-year-old woman with NF2 presented with a complaint of bilateral progressive sensorineural hearing loss for 10 years, which was similar in both ears. In 2009, the hearing loss became severe and communication problems aggravated. In 2006 she had undergone to partial tumor resection for facial nerve preservation on the left side at another institution. After surgery, the treatment was complemented with gama-knife radiation. She scheduled an appointment with our team seeking for a treatment for hearing rehabilitation. The tumor on her right side measured $1.5 \mathrm{~cm}$ (Figure 1). 
Due to the size of the tumor we believed it would be more convenient to attempt hearing preservation in the right ear and to try a cochlear implant. After counseling she agreed with and accepted surgery. In March 2010, the patient underwent retrolabyrinthine approach with complete tumor resection and anatomic preservation of the cochlear and facial nerves.

The retrolabyrinthine approach was performed with the patient placed in the classic supine position with the head rotated away from the surgeon. A retroauricular incision was made three centimeters posterior to the auricular sulcus extending to the mastoid tip. A muscle flap was placed anteriorly, allowing for the posterior retraction of the periosteum in order to drill the place into which the receiver/stimulator was lodged. A wild mastoidectomy was performed, exposing the entire posterior fossa dura mater, the sigmoid sinus and the jugular bulb. The posterior semicircular canal was partially drilled and obliterated, and the meatal plane was skeletonized. The vestibule was not opened. The posterior dura was opened from the base of the IAM to the anterior edge of the jugular bulb, extending to the edge of the sigmoid sinus. After cisternal drainage of cerebral spinal fluid the meatal plane was longitudinally opened and the tumor removed (Figure $2 \mathrm{~A}$ ).

The functional assessment of the cochlear nerve was evaluated through electrical promontory stimulation, which demonstrated present responses with poor morphology an unreliable reproducibility. Subsequently, cochlear implantation of a Nucleus 24RE Contour Advance
(Cochlear Ltd., Lane Cove, Australia) through the round window was performed. The electrode array was partially inserted (6 active electrodes out) (Figures 2 B, 3). Impedances of electrodes measurements were adequate. The patient had absent intraoperative neural response telemetry (NRT) in Electrodes 1, 22, 11, 6 and 16 with 25 $\mathrm{\mu s}$ (Figure $4 \mathrm{~A}$ ) and also in electrodes 11 and 15 with $50 \mu$ s (Figure $4 \mathrm{~B}$ ). No complications or additional deficts occured.

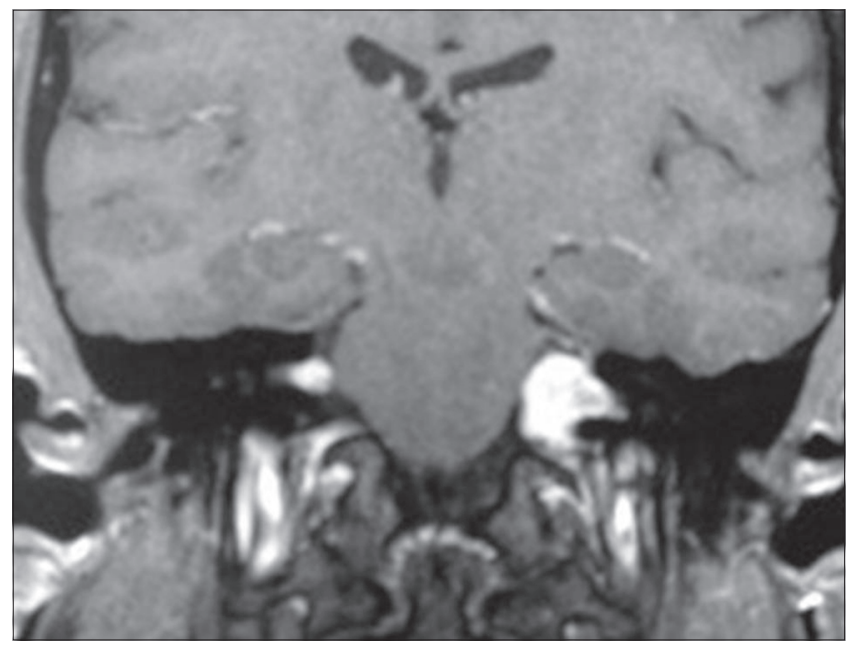

Figure 1. Coronal magnetic resonance imaging of the temporal bone showing bilateral cerebellopontine angle tumors with homogeneous enhancement after gadolinium injection on T1-weighted sequence, measuring $1.5 \mathrm{~cm}$ on the right side and $3.0 \mathrm{~cm}$ on the left side.

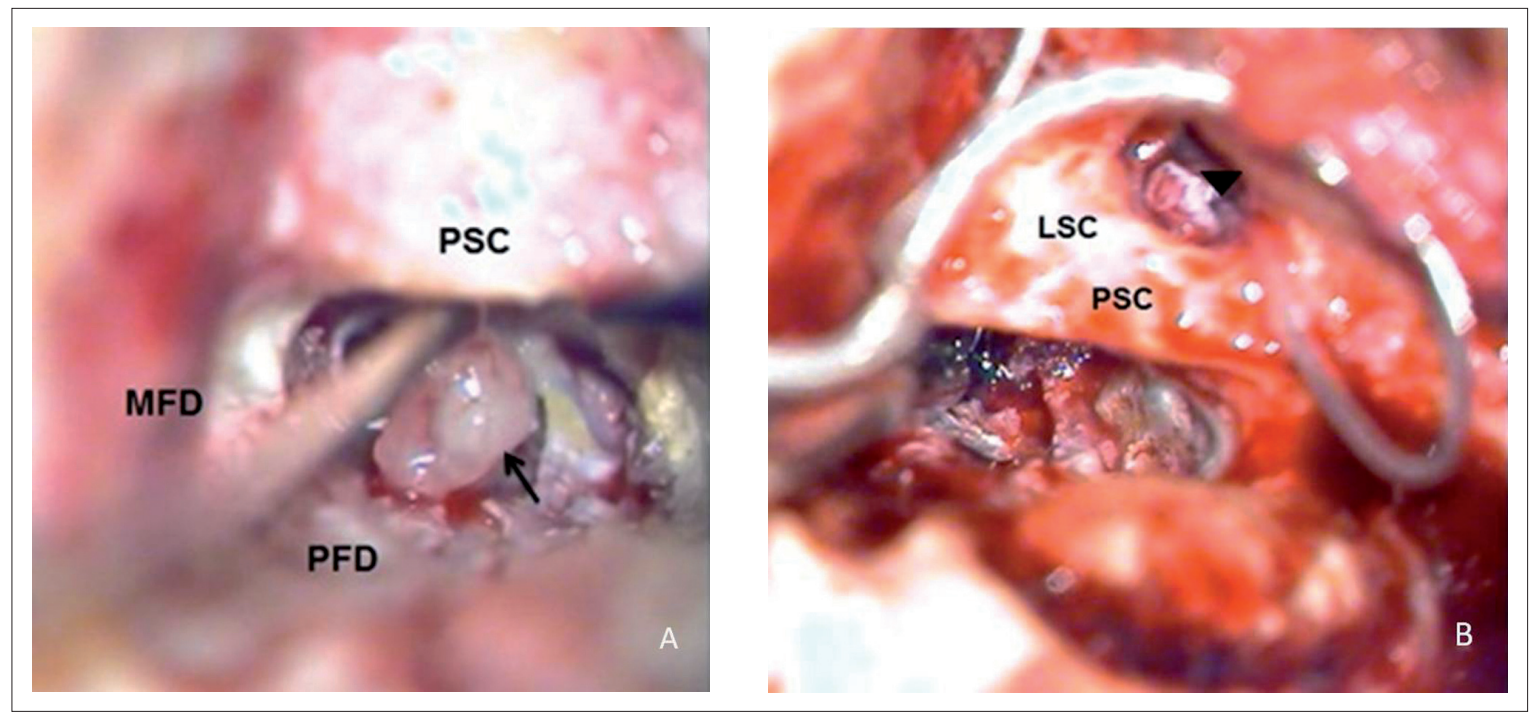

Figure 2.View of the retrolabyrinthine approach with vestibular schwannoma being removed (arrow) (A). View of the posterior tympanotomy approach with electrode array inserted in the coclea via round windown (arrow head) (B). (MFD, middle fossa dura-mater; PFD, posterior fossa dura-mater; PSC, posterior semi-circular canal; LSC, lateral semi-circular canal). 

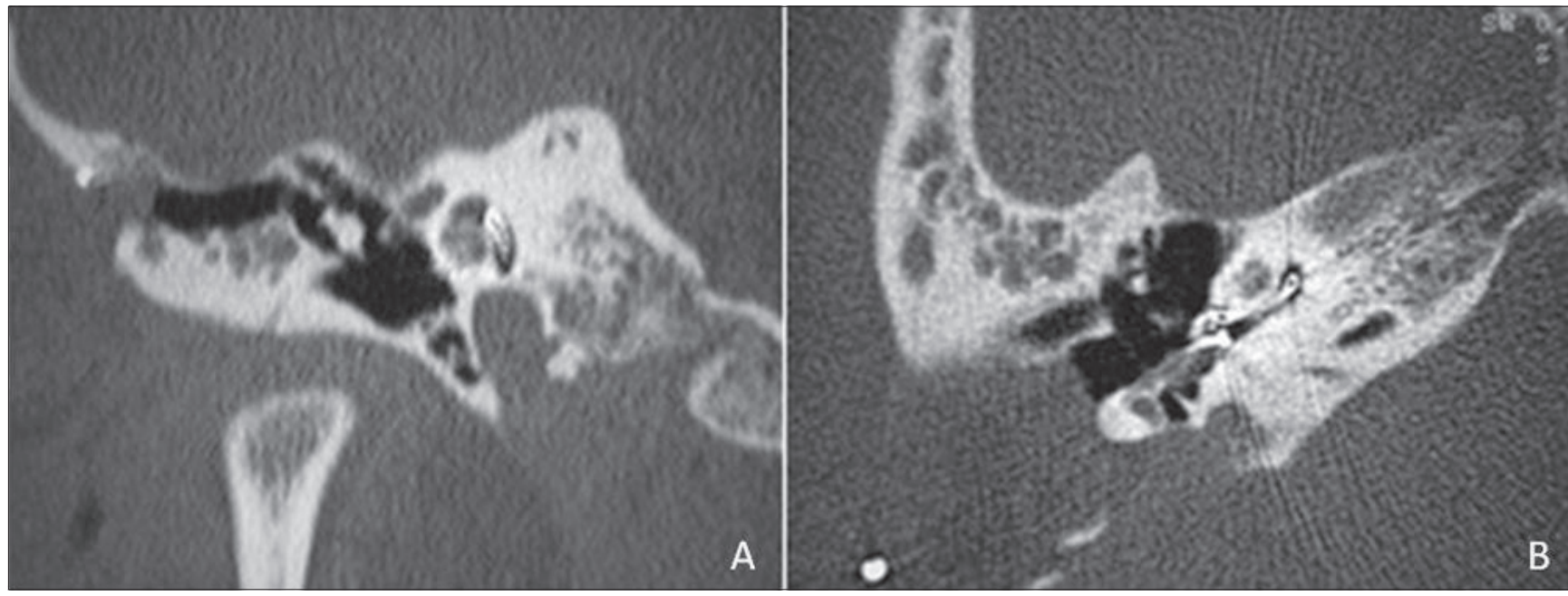

Figure 3. High-resolution coronal (A) and axial (B) CT scans of the temporal bones showing insertion of the electrode array into the middle and basal turns of the cochlea.

The cochlear implant was activated four weeks after surgery, with a Freedom speech processor with hearing sensation in 5 electrodes with advanced combination encoders (ACE) speech coding strategy $900 \mathrm{~Hz}$ of stimulation rate and 5 maxima, with 75 us pulse width. All other electrodes elicited visible facial nerve stimulation. Two months after activation she was referred to auditory training with a speech pathologist. With the

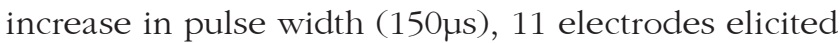
auditory response with very little or no facial stimulation. Sound field hearing threshold improvement was achieved (Figure 5). The mean tonal threshold was $46.2 \mathrm{dBHL}$. Despite the auditory training and the improvement in hearing thresholds, the patient could only detect environmental sounds and human voice but cannot discriminate vowels, words nor do sentences at 2 years of follow-up.

\section{DISCUSSION}

Cueva et al. (5) elicited auditory sensation through electrical promontory stimulation in 6 patients with NF2 who underwent tumor removal with cochlear nerve preservation. Since then, about 31 cases of cochlear implantation in NF2 patients have been reported in the literature (6-16). The timing of implantation varied from simultaneously to 10 years after tumor resection. Regardless of the time of cochlear implantation or the approach used, these studies have reported a diverse range of speech perception outcomes, from no benefit to significantly better speech discrimination (14).

The previously described approaches for $\mathrm{CI}$ in these patients were translabyrinthine, retrosigmoid or

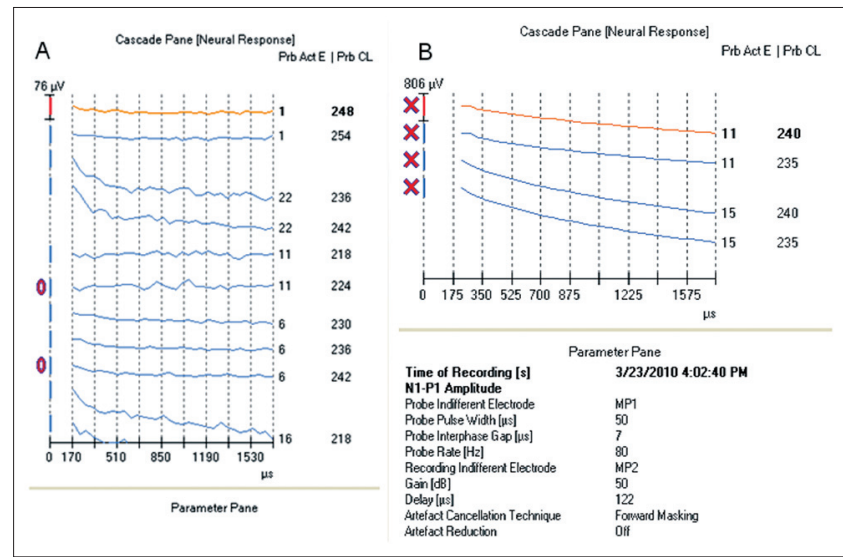

Figure 4. Intra-operative NRT showing absent evoked potentials on electrodes $1,22,11,6$, and 16 at 25 - $\mu$ s pulse width (A) and on electrodes 11 and 15 at $50-\mu$ s pulse width (B).

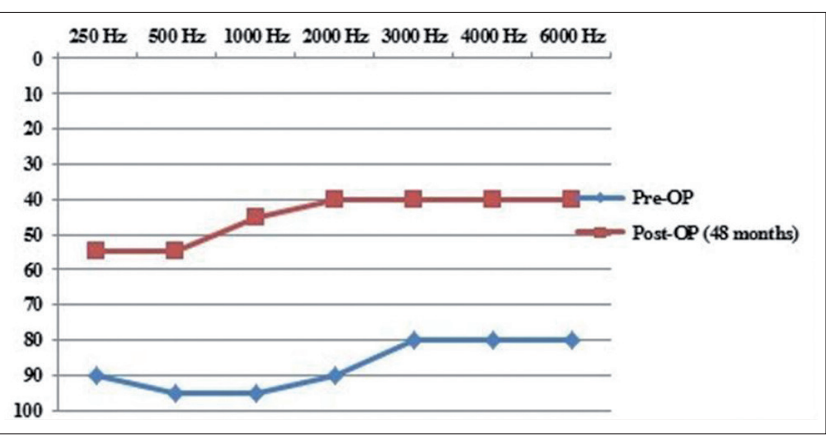

Figure 5. Sound field hearing threshold before and 48 months after surgery with the cochlear implant in the right ear. 
middle cranial fossa. The translabyrinthine approach for schwannoma removal is a highly destructive method, and degenerative changes in the cochlea such as fibrosis or ossification are more pronounced and occur in a short time (13). Thus, we hypothesized that the RLA with simultaneous cochlear implantation could bring better results.

Cochlear implantation offers advantages over ABI as reliable tonotopic stimulation of the auditory system. It is also less technically demanding and reduces the risk of major complications (16). As an attempt to avoid the overall poor hearing results of $\mathrm{ABI}$ and to limit the adverse effects of direct brainstem stimulation, cochlear implantation has been proposed and successfully performed in this case.

Surgical tumor resection may result in mechanical or thermal injury to the cochlear nerve or labyrinthine artery and traction on the eighth nerve may cause avulsion of delicate auditory fibers at the habenula perforata (16). Some studies demonstrate that hearing preservation is achieved in only $32 \%-67 \%$ of cases (16). Nevertheless, more than $80 \%$ of such patients are left with an anatomically intact auditory nerve (16). Thus, although anatomic conservation of the cochlear nerve is fundamental, if the functional preservation is not achieved, the auditory results may be poor. The use of electrical promontory stimulation testing is controversial as positivity does not guarantee cochlear implant performance. Our patient's test showed present responses and cochlear implant was performed with improvement in hearing thresholds, but she cannot discriminate speech.

\section{CONCLUSION}

Cochlear implantation is a feasible auditory restoration option in NF2 when cochlear anatomic and functional nerve preservation is achieved. The RLA approach was adequate for this purpose and presents an option for hearing preservation in NF2 patients.

\section{REFERENCES}

1. Kanowitz SJ, Shapiro WH, Golfinos JG, Cohen NL, Roland JT Jr. Auditory brainstem implantation in patients with neurofibromatosis type 2. Laryngoscope. 2004;114:213546.

2. Vincenti V, Pasanisi E, Guida M, Di Trapani G, Sanna M. Hearing rehabilitation in neurofibromatosis type 2 patients: cochlear versus auditory brainstem implantation. Audiol Neurootol. 2008;13:273-80.
3. Hitselberger WE, House WF, Edgerton BJ, Whitaker S. Cochlear nucleus implants. Otolaryngol Head Neck Surg. 1984;92(1):52-4.

4. Tran Ba Huy P, Kania R, Frachet B, Poncet C, Legac MS. Auditoryrehabilitation with cochlear implantation in patients with neurofibromatosis type 2.Acta Otolaryngol. 2009;129(9):971-5.

5. Cueva RA, Thedinger BA, Harris JP, Glasscock ME 3rd. Electrical promontory stimulation in patients with intact cochlear nerve and anacusis following acoustic neuroma surgery. Laryngoscope. 1992;102:1220-24.

6. Tono T, Ushisako Y, Morimitsu T: Cochlear implantation in an intralabyrinthine acoustic neuroma patient after resection of an intracanalicular tumor. J Laryngol Otol. 1996;110:570-73.

7. Graham J, Lynch C, Weber B, Stollwerck L, Wei J, Brookes G: The magnetless Clarion cochlear implant in a patient with neurofibromatosis 2. J Laryngol Otol. 1999;113:45863.

8. Temple RH, Axon PR, Ramsden RT, Keles N, Deger K, Yucel E: Auditory rehabilitation in neurofibromatosis type 2: a case for cochlear implantation. J Laryngol Otol. 1999;113:161-63 .

9. Ahsan S, Telischi F, Hodges A, Balkany T: Cochlear implantation concurrent with translabyrinthine acoustic neuroma resection. Laryngoscope. 2003;113:472-74.

10. Nolle C, Todt I, Basta D, Unterberg A, Mautner VF, Ernst A: Cochlear implantation after tumor resection in neurofibromatosis type 2: impact of infra-and postoperative neural response telemetry monitoring. ORL. 2003;65:23034 .

11. Aristegui M, Denia A: Simultaneous cochlear implantation and translabyrinthine removal of vestibular schwannoma in an only hearing ear: report of two cases (neurofibromatosis type 2 and unilateralvestibular schwannoma). Otol Neurotol. 2005;26:205-10.

12. Lustig LR, Yeagle J, Driscoll CL, Blevins N, Francis H, Niparko JK. Cochlear implantation in patients with neurofibromatosis type 2 and bilateral vestibular schwannoma. Otol Neurotol. 2006;27:512-18.

13. Vincenti V, Pasanisi E, Guida M, Di Trapani G, Sanna M. Hearing rehabilitation in neurofibromatosis type 2 patients: cochlear versus auditory brainstem implantation. Audiol Neurootol. 2008;13:273-80. 
14. Celis-Aguilar E, Lassaletta L, Gavilán J. Cochlear implantationin patients with neurofibromatosis type 2 and patients with vestibular schwannoma in the only hearing ear. Int J Otolaryngol. 2012;2012:157497.

15. Belal A. Is cochlear implantation possible after acoustic tumor removal?Otol Neurotol. 2001;22(4):497-500.
16. Carlson ML, Breen JT, Driscoll CL, Link MJ, Neff BA, Gifford $\mathrm{RH}$, Beatty CW. Cochlear implantation in patients with neurofibromatosis type 2: variables affecting auditory performance. Otol Neurotol. 2012;33(5):853-62. 